\title{
Observational constraints on models for the interstellar magnetic field in the Galactic disk
}

\author{
H. Men ${ }^{1,2}$, K. Ferrière ${ }^{2}$, and J. L. Han ${ }^{1}$ \\ 1 National Astronomical Observatories, Chinese Academy of Sciences, Jia 20 DaTun Road, Chaoyang District, Beijing 100012, \\ PR China \\ e-mail: ferriere@ast.obs-mip.fr; mh@bao.ac.cn \\ 2 Observatoire Midi-Pyrénées, Université Paul Sabatier Toulouse 3, CNRS, 14 Av. Ed. Belin, 31400 Toulouse, France
}

Received 17 September 2007 / Accepted 8 May 2008

ABSTRACT

\begin{abstract}
Aims. Our purpose is to place firm observational constraints on the three most widely used theoretical models for the spatial configuration of the large-scale interstellar magnetic field in the Galactic disk, namely, the ring, the axisymmetric, and the bisymmetric field models.

Methods. We used the rotation measures (RMs) of low-latitude Galactic pulsars and combined them with their dispersion measures and estimated distances to map out the line-of-sight component of the interstellar magnetic field in the near half of the Galactic disk. We then fit our map of the line-of-sight field to the three aforementioned theoretical field models and discuss the acceptability of each fit, in order to determine whether the considered field model is allowed by the pulsar data or not.

Results. Strictly speaking, we find that all three field models are ruled out by the pulsar data. Furthermore, none of them appears to perform significantly better than the others. From this we conclude that the large-scale interstellar magnetic field in the Galactic disk has a more complex pattern than just circular, axisymmetric, or bisymmetric.
\end{abstract}

Key words. ISM: magnetic fields - Galaxy: disk - galaxies: magnetic fields

\section{Introduction}

The interstellar magnetic field of our Galaxy has been the object of intense investigation since the early 1980s. Different observational methods (e.g., based on synchrotron emission, Faraday rotation, Zeeman splitting, polarization of starlight, polarization of dust infrared emission) provide information on the magnetic field in different interstellar regions. Faraday rotation of Galactic pulsars and extragalactic linearly polarized radio sources make it possible to directly trace the magnetic field in ionized regions. In practice, one measures the so-called rotation measure (RM), defined by

$\mathrm{RM}=0.81 \int_{0}^{d} n_{\mathrm{e}} B_{\|} \mathrm{d} s \operatorname{rad~m}^{-2}$,

where $n_{\mathrm{e}}$ is the free-electron density (in $\mathrm{cm}^{-3}$ ), $B_{\|}$is the lineof-sight component of the magnetic field (in $\mu \mathrm{G}$ ) and $d$ is the distance to the radio source (in pc). Pulsars present a number of advantages when used as probes of the interstellar magnetic field. In particular, they are highly linearly polarized, they have no intrinsic rotation measure and their distances can be estimated reasonably well. Moreover, the RM of a pulsar divided by its dispersion measure (DM),

$\mathrm{DM}=\int_{0}^{d} n_{\mathrm{e}} \mathrm{d} s \quad \mathrm{~cm}^{-3} \mathrm{pc}$,

directly yields the $n_{\mathrm{e}}$-weighted average value of $B_{\|}$along its line of sight,

$\overline{B_{\|}}=1.232 \frac{\mathrm{RM}}{\mathrm{DM}} \mu \mathrm{G}$.
We now know that the interstellar medium (ISM) is highly inhomogeneous and that the interstellar magnetic field has an important turbulent component. For this reason, neighboring pulsars may have significantly different values of RM and DM, and a plot RM versus DM will generally exhibit a large scatter. However, if one considers a Galactic region larger than the scale of the turbulent field and containing enough pulsars for statistical purposes, one can infer the large-scale (or regular) component of $\overline{B_{\|}}$in that region from the slope of the mean DM-RM relation (Rand \& Lyne 1994):

$$
\left\langle\overline{B_{\|}}\right\rangle=1.232\left\langle\frac{\mathrm{d} \mathrm{RM}}{\mathrm{d} \mathrm{DM}}\right\rangle \mu \mathrm{G}
$$

Various theoretical models have been proposed to describe the spatial structure of the large-scale magnetic field in the Galaxy. First and foremost are the ring model, the axisymmetric or axisymmetric spiral (ASS) model, and the bisymmetric or bisymmetric spiral (BSS) model. According to the galactic dynamo theory, ASS fields would be easiest to amplify under typical galactic conditions (e.g., Ruzmaikin et al. 1985; Ferrière \& Schmitt 2000), whereas BSS fields could possibly be excited in the presence of an external disturbance, such as a companion galaxy (Moss 1995, 1996). On the other hand, the primordial field theory naturally leads to BSS fields (Howard \& Kulsrud 1997).

In principle, RM studies are ideally suited to establish the overall structure of the Galactic magnetic field. However, the different RM studies performed so far yield contradictory results: some favor a ring field (Rand \& Kulkarni 1989; Rand \& Lyne 1994; Vallée 2005), others an axisymmetric or ASS field (Vallée 1991, 1996), and others a bisymmetric or 


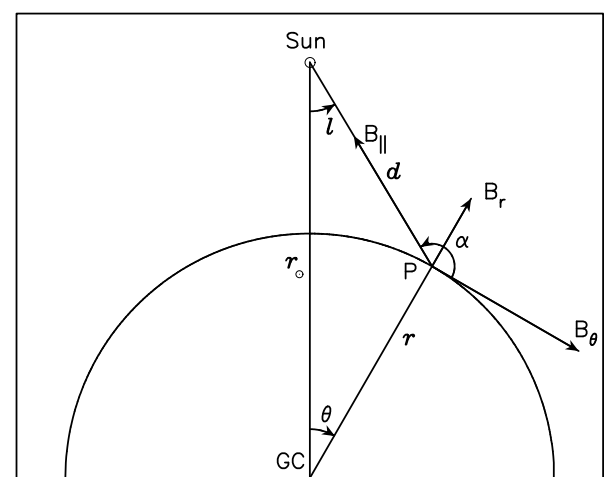

Fig. 1. Schematics showing the geometrical variables associated with a pulsar P (see main text for the exact definitions).

BSS field (Simard-Normandin \& Kronberg 1980; Han \& Qiao 1994; Indrani \& Deshpande 1999; Han et al. 2006). Moreover, although all these studies conclude with a preferred field model, none of them has seriously considered the possibility that more than one model is allowed by the RM data or, alternatively, that none of the three basic models alone can account for the data. Hence the question we would like to address in this paper: which among the ring, axisymmetric and bisymmetric models can clearly be accepted on the grounds that it is consistent with the RM data, and which model should clearly be rejected on the grounds that it fails to provide a good fit to the data.

In recent years, numerous pulsars were discovered in the near half of the Galactic disk and many of them had their RM measured. At the present time, among the $\sim 1800$ known pulsars, 690 have measured RMs and, among the latter, 524 are located at low Galactic latitudes $\left(|b|<10^{\circ}\right)$. Pulsars with measured RMs now provide a reasonably good coverage of the near half of the Galactic disk. Furthermore, pulsar distances can now be estimated with fairly good accuracy thanks to the improved free-electron density model of Cordes \& Lazio (2002) (known as the NE2001 model). The new measurements enable one to investigate the configuration of the Galactic magnetic field over a much larger region and with much more confidence than previously feasible.

In Sect. 2, we present the three basic theoretical models for the interstellar magnetic field in the Galactic disk. In Sect. 3, we describe the procedure used to bin the pulsar data and to map out the distribution of $B_{\|}$. In Sect. 4, we fit our map of $B_{\|}$to each of the three field models, and we discuss how good the fits are at reproducing the pulsar data. In Sect. 5, we summarize our results and conclude our study.

\section{Description of the field models}

Throughout this paper, the Galactocentric cylindrical coordinates are denoted by $(r, \theta, z)$, and the distance from the Galactic center (GC) to the Sun is set to $r_{\odot}=8.5 \mathrm{kpc}$.

In general, the horizontal position of a given pulsar $\mathrm{P}$ can be defined either by its distance from the GC, $r$, and its Galactic azimuthal angle $\theta$ (which increases clockwise from $\theta=0$ along the line segment GC-Sun), or by its distance from the Sun, $d$, and its Galactic longitude, $l$ (which increases counterclockwise from $l=0$ along the line segment Sun-GC). Another useful angular coordinate is the angle $\alpha$ between the azimuthal direction at $\mathrm{P}$ and the vector P-Sun, such that $\alpha=\theta+l+\frac{\pi}{2}$ (see Fig. 1).

Because Galactic differential rotation efficiently stretches magnetic field lines in the azimuthal direction, $\left\langle B_{\theta}\right\rangle$ dominates over both $\left\langle B_{r}\right\rangle$ and $\left\langle B_{z}\right\rangle$. Moreover, all the pulsars selected for the present work lie at $|b|<10^{\circ}$ and reside in the Galactic disk. There, the large-scale magnetic field is nearly horizontal (e.g., Ruzmaikin et al. 1985; Beck et al. 1996), so that $\left|\left\langle B_{z}\right\rangle\right| \ll$ $\left|\left\langle B_{r}\right\rangle\right|,\left|\left\langle B_{\theta}\right\rangle\right|$. In addition, projecting an already small $\left\langle B_{z}\right\rangle$ onto the line of sight to a pulsar further reduces its contribution by a factor $|\sin b| \ll 1$. Under these conditions, the line-of-sight component of the large-scale magnetic field depends only on its radial and azimuthal components, and is related to them through

$\left\langle B_{\|}\right\rangle=\left\langle B_{r}\right\rangle \sin \alpha+\left\langle B_{\theta}\right\rangle \cos \alpha$.

We now present the three theoretical field models.

\subsection{Ring model}

In the ring model, the large-scale magnetic field points everywhere in the azimuthal direction, so that its radial component vanishes:

$\left\langle B_{r}\right\rangle=0$.

Its azimuthal component is constant along circles, i.e., independent of $\theta$, but it can vary with $r$ :

$\left\langle B_{\theta}\right\rangle=\left\langle B_{\theta}\right\rangle(r)$,

and it can even change sign along a Galactic radius. As a matter of fact, all RM studies leading to a ring model have found reversals in $\left\langle B_{\theta}\right\rangle$ (Rand \& Kulkarni 1989; Rand \& Lyne 1994; Vallée 2005). It should be noted that the ring model constitutes a particular case of the axisymmetric model.

\subsection{Axisymmetric model}

In the axisymmetric model, $\left\langle B_{r}\right\rangle$ and $\left\langle B_{\theta}\right\rangle$ are both independent of $\theta$ and vary only with $r$ :

$\left\langle B_{r}\right\rangle=\left\langle B_{r}\right\rangle(r)$,

$\left\langle B_{\theta}\right\rangle=\left\langle B_{\theta}\right\rangle(r)$.

Here, too, $\left\langle B_{\theta}\right\rangle$ can reverse sign with $r$. Such sign reversals were found in RM studies favoring an ASS magnetic field (Vallée 1991, 1996). Interestingly, reversals in $\left\langle B_{\theta}\right\rangle$ are also consistent with dynamo theory, which can produce them under certain conditions, e.g., when the magneto-ionic disk has a particular shape and thickness and the seed field itself has strong reversals (Poezd et al. 1993) or when the Galactic rotation rate decreases not only with radius but also with height (Ferrière \& Schmitt 2000).

\subsection{Bisymmetric model}

In the bisymmetric model, $\left\langle B_{r}\right\rangle$ and $\left\langle B_{\theta}\right\rangle$ have a simple sinusoidal dependence on $\theta$, which can be written in the form (Berkhuijsen et al. 1997):

$$
\begin{aligned}
& \left\langle B_{r}\right\rangle=b_{r}(r) \sin (\theta-\phi(r)), \\
& \left\langle B_{\theta}\right\rangle=b_{\theta}(r) \sin (\theta-\phi(r)),
\end{aligned}
$$

where $b_{r}(r)$ and $b_{\theta}(r)$ are the maximum amplitudes of $\left\langle B_{r}\right\rangle$ and $\left\langle B_{\theta}\right\rangle$, respectively, and $\phi(r)$ is the azimuthal phase. Both $\left\langle B_{r}\right\rangle$ and $\left\langle B_{\theta}\right\rangle$ can reverse sign with $r$. The magnetic pitch angle is defined as

$\tan p(r)=\frac{\left\langle B_{r}\right\rangle}{\left\langle B_{\theta}\right\rangle}=\frac{b_{r}(r)}{b_{\theta}(r)} ;$

it is positive (negative) if the magnetic field spirals out clockwise (counterclockwise) or spirals in counterclockwise (clockwise). 
(a)

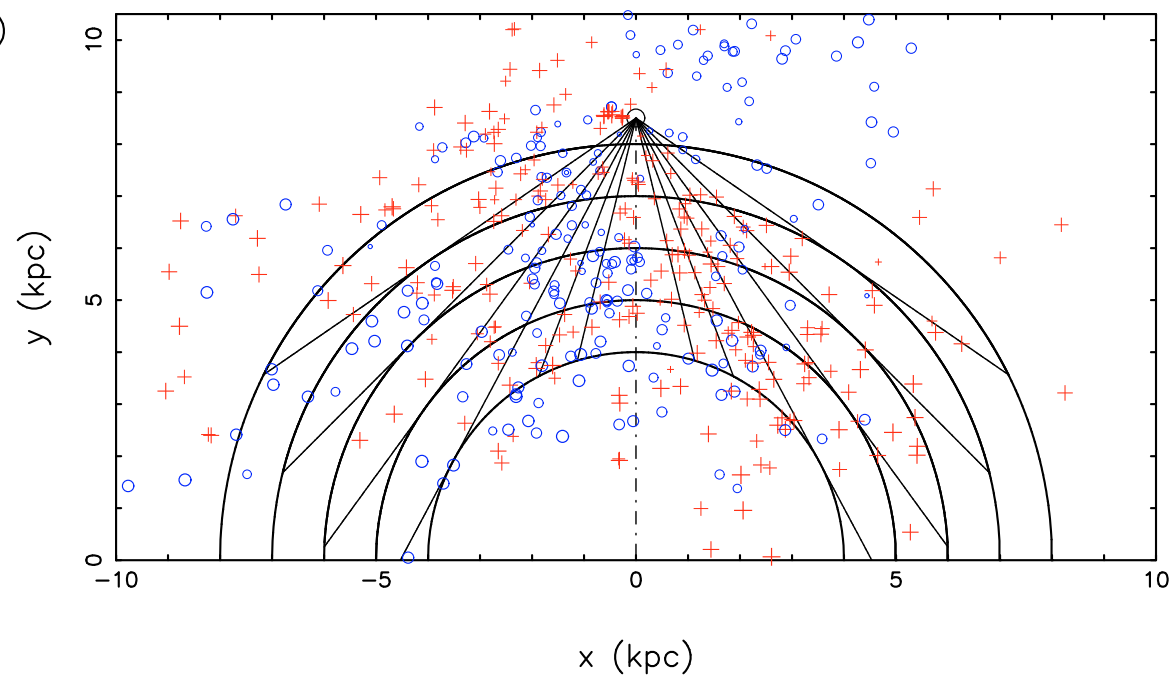

(b)



Fig. 2. Grids used in our study overlaid on the face-on distribution of our 482 low-latitude $\left(|b|<10^{\circ}\right.$ ) pulsars. Pulsars with a positive (negative) $\mathrm{RM}$ are denoted with crosses (circles). $(x, y)$ are Galactocentric cartesian coordinates. The Sun is located at $(x=8.5 \mathrm{kpc}, y=0)$. In the upper panel, the circles underlying the grids are at $r=4 \mathrm{kpc}, 5 \mathrm{kpc}, 6 \mathrm{kpc}, 7 \mathrm{kpc}$ and $8 \mathrm{kpc}$, while in the lower panel, they are at $r=3.5 \mathrm{kpc}, 4.5 \mathrm{kpc}, 5.5 \mathrm{kpc}, 6.5 \mathrm{kpc}, 7.5 \mathrm{kpc}$ and $8.5 \mathrm{kpc}$.

\section{Mapping of $\left\langle\overline{B_{\|}}\right\rangle$}

To date, there are 690 pulsars with measured RMs (Hamilton \& Lyne 1987; Rand \& Lyne 1994; Qiao et al. 1995; van Ommen et al. 1997; Han et al. 1999; Crawford et al. 2001; Mitra et al. 2003; Weisberg et al. 2004; Han et al. 2006; Noutsos et al. 2008). Among these pulsars, we selected those that lie at low Galactic latitudes $\left(|b|<10^{\circ}\right)$ and have reliable RMs (error on $\mathrm{RM}<25 \mathrm{rad} \mathrm{m}^{-2}$ ). This left us with 482 pulsars. For the distances and DMs of our selected pulsars, we used the values given in the ATNF Pulsar Catalog (Manchester et al. 2005, see http: //www . atnf.csiro.au/research/pulsar/ psrcat). Pulsar distances in this catalog were estimated with the help of Cordes \& Lazio's (2002) NE2001 model for the freeelectron density; for pulsars located in the inner Galaxy, individual distances are typically uncertain by $\sim 20 \%$, but the relative distances of neighboring pulsars have a much lower uncertainty. Pulsar DMs, for their part, are known with good accuracy (error on DM generally $<1 \mathrm{~cm}^{-3} \mathrm{pc}$ ).
In order to map out the large-scale component of $\overline{B_{\|}}$, one needs to divide the Galactic disk into regions (boxes) having sizes intermediate between the large scales of the regular field and the small scales of the turbulent field and containing at least a few pulsars each. In previous studies (Rand \& Lyne 1994; Weisberg et al. 2004; Han et al. 2006), this division was based on a heliocentric grid defined by circles of constant $d$ and radial lines of constant $l$. Such a heliocentric division was justified by the spatial distribution of the available pulsars, but it is ill-suited to the present work, whose purpose is to test field models expressed in terms of Galactic radius, $r$. A much more appropriate division here is one based on a hybrid grid defined by circles of constant $r$ and lines of constant $l$ (see Fig. 2). To make full use of the pulsar data, we consider two different grids. In the first grid, the circles are located at $r=4 \mathrm{kpc}, 5 \mathrm{kpc}, 6 \mathrm{kpc}, 7 \mathrm{kpc}$ and $8 \mathrm{kpc}$ (i.e., $r=r_{i}$, with $\left.r_{i} \equiv i \mathrm{kpc}, i=4 \ldots 8\right)$, and the lines of constant $l$ are the lines emanating from the Sun and tangent to one of the circles $r=r_{i}, i=2 \ldots 7$, plus the line Sun-GC (i.e., $l=l_{0}, l_{ \pm i}$, 
with $l_{0} \equiv 0$ and $l_{ \pm i} \equiv \pm \operatorname{asin} \frac{r_{i}}{r_{\odot}}, i=2 \ldots 7$ ) (see Fig. 2a). The second grid is defined in an analogous manner with the circles shifted by $0.5 \mathrm{kpc}$ (i.e., $r=r_{i}, i=3.5,4.5 \ldots 8.5$, and accordingly, $l=l_{0}, l_{ \pm i}, i=1.5,2.5 \ldots 7.5$ ) (see Fig. 2b). To ensure a sufficient number of pulsars per box, some of the boxes defined by these grids are paired together. More specifically, the nonoutermost boxes along each ring are paired either with their left or right neighbor along the same ring (thereby leading to a single double-size box) or with both neighbors separately (thereby leading to two overlapping boxes).

Altogether, we have 9 rings, which are centered on $r_{i} \equiv i \mathrm{kpc}$, with $i=4.5,5.5 \ldots 7.5$ in the first grid and $i=4,5 \ldots 8$ in the second grid. In the following, the ring centered on $r_{i}$ (and extending between $r_{i-0.5}$ and $r_{i+0.5}$ ) is referred to as ring $i$.

We retain only the boxes containing at least 5 RMs. This minimum number of RMs, which is smaller than generally recommended for statistical testing, results from the limited number of pulsars with know RMs; it was chosen as a trade-off between the need to have enough data points per box to make use of Eq. (4) and the need to have enough boxes on the Galactic plane to capture the spatial variations of the large-scale magnetic field. Even with such a small number of RMs in a given box, it is possible to trace the dependences of RM on distance and on DM for the pulsars lying in this box (see Rand \& Lyne 1994; Weisberg et al. 2004; Han et al. 2006).

We estimate the average value of $\overline{B_{\|}}$, denoted by $\left\langle\overline{B_{\|}}\right\rangle$, in each of the retained boxes in the following way: we plot the points (DM, RM) of all the pulsars in the box, we fit a straight line through the resulting set of data points, and we take $\left\langle\overline{B_{\|}}\right\rangle$to be 1.232 times the slope of this line (see Eq. (4) and preceding comment). To perform the straight-line fit, we resort to a slightly modified version of the ordinary least-squares linear regression of $Y$ on $X$ described by Isobe et al. (1990), which is well suited when the dispersion of the data points about the linear relation cannot be calculated beforehand. The modifications brought to the original scheme are designed to exclude the occasional outliers - such as those arising from HII regions (Mitra et al. 2003). In practice, we discard all the data points whose absolute deviation from the mean RM or DM exceeds three times the mean absolute deviation. To illustrate the procedure, we show two examples in Fig. 3.

The derived values of $\left\langle\overline{B_{\|}}\right\rangle$in all the boxes of our two grids are mapped in Fig. 4. For convenience, these values are converted into vectors oriented along the local line of sight.

As explained above Eq. (4), the turbulent component of the magnetic field, $\delta \boldsymbol{B}$, causes the RMs to scatter about the mean DM-RM line. This physical scatter due to turbulence is typically one order of magnitude larger than the observational scatter due to measurement errors. The amplitude of the RM scatter is given by the rms deviation of the measured RMs from the mean DM-RM line. Since the RM scatter is of predominantly turbulent origin, its amplitude divided by the mean DM in the considered box directly yields (to a factor 1.232) an estimate for the rms value of the turbulent component of the line-of-sight field, $\delta \overline{B_{\|}}$. Finally, the rms value of $\delta \overline{B_{\|}}$divided by the square root of the number of pulsars in the box provides an estimate for the statistical uncertainty in $\left\langle\overline{B_{\|}}\right\rangle, \sigma_{\|}$, which, again, is predominantly due to turbulence. The exact expression of $\sigma_{\|}$can be found in Isobe et al. (1990). With our data, the typical values of $\sigma_{\|}$lie between $\sim 0.2 \mu \mathrm{G}$ and $1.3 \mu \mathrm{G}$.
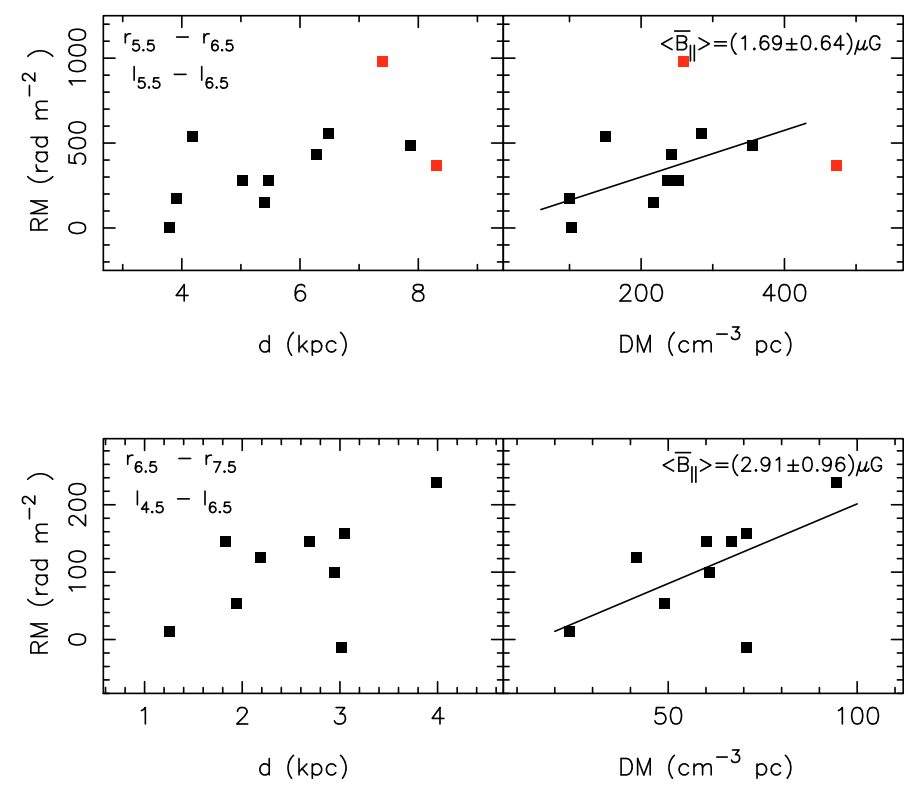

Fig. 3. Plots of RM versus distance (left panels) and versus DM (right panels) for the pulsars lying in two different boxes. The first box (top row) is delimited by the circles $r=r_{5.5}$ and $r=r_{6.5}$ in the radial direction and by the tangential lines $l=l_{5.5}$ and $l=l_{6.5}$ in the longitudinal direction. The second box (bottom row) is delimited by the circles $r=r_{6.5}$ and $r=r_{7.5}$ and by the tangential lines $l=l_{4.5}$ and $l=l_{6.5}$. For each box, the best-fit straight line through the points (DM, RM) is drawn in the right panel, and the corresponding value of $\left\langle\overline{B_{\|}}\right\rangle$with its statistical uncertainty are written in the upper right corner. The red points represent outliers.

\section{Data fitting to the field models}

Once we have obtained a set of observational values of $\left\langle\overline{B_{\|}}\right\rangle$ together with their statistical uncertainties (or error bars), we can put the three theoretical field models presented in Sect. 2 to the test. As in all other studies based on RMs, we proceed on the notion that the large-scale interstellar magnetic field may be identified with its $n_{\mathrm{e}}$-weighted average value (denoted with an overbar). Implicit here is the assumption that fluctuations in magnetic field strength and in free-electron density are statistically uncorrelated. In reality, this assumption is certainly not strictly satisfied in the ISM (e.g., Beck et al. 2003), and this will cause our results to be somewhat biased.

With this caveat in mind, we now describe the overall procedure. For each model, we use all our observational values of $\left\langle\overline{B_{\|}}\right\rangle$to derive the best-fit parameters of the model. We then examine whether the best fit is consistent with the pulsar data, i.e., whether the theoretical line-of-sight fields predicted by it fall within the error bars of the observational $\left\langle\overline{B_{\|}}\right\rangle$(in a statistical sense). If we find that the best fit is not consistent with the data, we may conclude that the considered model must be rejected. If, on the other hand, the best fit is found consistent with the data, we may conclude that the model is acceptable; we then determine the extent of the so-called "consistency domain", i.e., the parameter domain around the best fit within which solutions are consistent with the data.

It is important to realize that the concept of acceptability differs from the concept of detectability. A given field model, say, the ring model, is acceptable only if it is not ruled out by the available pulsar data. This does not necessarily imply that the Galactic magnetic field is really of the ring type, nor that a ring 


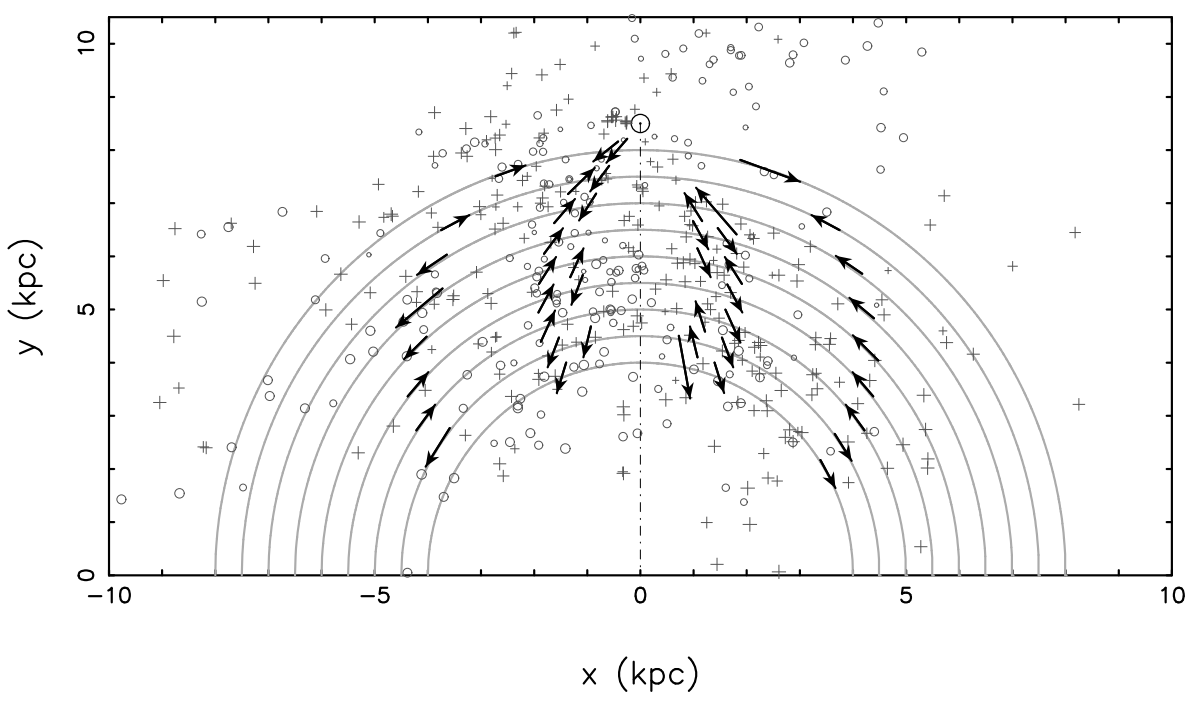

Fig. 4. Face-on map showing the average line-of-sight component of the magnetic field, $\left\langle\overline{B_{\|}}\right\rangle$, obtained in the different boxes of our two grids. Each $\left\langle\overline{B_{\|}}\right\rangle$is plotted in the form of a vector centered on the box midpoint (point at middle radius, $r=r_{i}$, and middle longitude, $l=\left(l_{\min }+l_{\max }\right) / 2$ ), and oriented along the local line of sight.

field has truly been detected. Detection of a ring field requires not only that the ring model be acceptable, but also that the zerofield solution do not belong to the consistency domain.

Let us now discuss more specifically what exact criterion should be used to test consistency with the pulsar data for a given field model. Each of the three models is characterized by a number of independent free functions of Galactic radius $\left(\left\langle B_{\theta}\right\rangle(r)\right.$ in the ring model; $\left\langle B_{r}\right\rangle(r)$ and $\left\langle B_{\theta}\right\rangle(r)$ in the axisymmetric model; $b_{r}(r), b_{\theta}(r)$ and $\phi(r)$ in the bisymmetric model), corresponding to the same number of independent free parameters in every ring $i$ (denoted by $\left\langle B_{\theta}\right\rangle_{i}$ in the ring model; $\left\langle B_{r}\right\rangle_{i}$ and $\left\langle B_{\theta}\right\rangle_{i}$ in the axisymmetric model; $b_{r, i}, b_{\theta, i}$ and $\phi_{i}$ in the bisymmetric model). Therefore, the 9 different rings may be analyzed separately.

For any one of the three field models, consider a given ring $i$ and suppose that this ring contains $n_{i}$ boxes. For every box $j$, we have derived an observational value of the average line-of-sight field $\left\langle\overline{B_{\|}}\right\rangle$, denoted by $\left\langle\overline{B_{\|}}\right\rangle_{i j}$, together with its statistical uncertainty, denoted by $\left(\sigma_{\|}\right)_{i j}$. Besides, we can calculate a theoretical expression of the large-scale line-of-sight field $\left\langle B_{\|}\right\rangle$, denoted by $\left\langle B_{\|}\right\rangle_{i j}$, in terms of the free parameters of ring $i$. The best-fit values of these parameters are obtained by minimizing

$\chi^{2}=\sum_{j=1}^{n_{i}}\left(\frac{\left\langle\overline{B_{\|}}\right\rangle_{i j}-\left\langle B_{\|}\right\rangle_{i j}}{\left(\sigma_{\|}\right)_{i j}}\right)^{2}$.

The best fit of ring $i$ can be considered consistent with the pulsar data if, on average over ring $i$, the theoretical best-fit $\left\langle B_{\|}\right\rangle_{i j}$ do not differ from the observational $\left\langle\overline{B_{\|}}\right\rangle_{i j}$ by more than the associated uncertainties $\left(\sigma_{\theta}\right)_{i j}$. In mathematical terms, this condition for consistency can be expressed as $\chi^{2} \leq n_{i}$. However, when the number of data points, $n_{i}$, is not much greater than the number of free parameters, $v(v=1$ for the ring model; $v=2$ for the axisymmetric model; $v=3$ for the bisymmetric model), consistency with the data should be tested with the more exact criterion

$\chi^{2} \leq n_{i}-v$,

where $n_{i}-v$ is the number of degrees of freedom, i.e., the number of data points that cannot automatically be placed on a curve with $v$ adjustable parameters. Equation (14) provides a rule of thumb for a reasonably good fit (see Sect. 15.1 in Press et al. 1992). If $\chi^{2} \gg n_{i}-v$, the best-fit curve misses too many data points to be believable.

It is possible to obtain a more rigorous (and, at the same time, more flexible) criterion for consistency. Suppose, for the sake of argument, that the model we are testing is correct. If the data points $\left\langle\overline{B_{\|}}\right\rangle_{i j}$ of ring $i$ follow a Gaussian distribution, $\chi^{2}$ has a chi-square distribution with $n_{i}-v$ degrees of freedom. One can then calculate the a priori probability, $P\left[\chi^{2}>\chi_{\text {crit }}^{2}\right.$, that the $\chi^{2}$ obtained for a particular set of data points exceeds some critical value $\chi_{\text {crit }}^{2}$. Conversely, one can calculate the critical $\chi_{\text {crit }}^{2}$ for which $P\left[\chi^{2}>\chi_{\text {crit }}^{2}\right]$ equals some imposed probability $P_{0}$. For instance, if the model is correct, it is unlikely (only $10 \%$ chance) that $\chi^{2}>\chi_{\text {crit }}^{2}\left(P_{0}=0.1\right)$. Turning the statement around, if we find $\chi^{2}>\chi_{\text {crit }}^{2}\left(P_{0}=0.1\right)$, it is unlikely that the model is correct we will say that the model is inconsistent with the data. This reasoning directly leads to the following consistency condition:

$\chi^{2} \leq \chi_{\text {crit }}^{2}\left(P_{0}\right)$

Here, we will adopt $P_{0}=0.1$ as our default value, but we will also discuss the results obtained for $P_{0}=0.05$. In practice, the values of $P\left[\chi^{2}>\chi_{\text {crit }}^{2}\right]$ for given $n_{i}-v$ and $\chi_{\text {crit }}^{2}$ are tabulated in various textbooks (e.g., Yamane 1964). The tables can also be used to determine $\chi_{\text {crit }}^{2}\left(P_{0}\right)$ for given $n_{i}-v$ and $P_{0}$. For reference, the values of $\chi_{\text {crit }}^{2}\left(P_{0}\right)$ for $n_{i}-v=1,2,3,4,5$ and for $P_{0}=$ $0.05,0.1,0.2$ are listed in Table 1 . An important point emerging from Table 1 is that $\chi_{\text {crit }}^{2}\left(P_{0}=0.1\right)>n_{i}-v$, so that Eq. (15) with $P_{0}=0.1$ will always be easier to satisfy than Eq. (14).

In the next three subsections, we present the results obtained with the rule of thumb (Eq. (14)) and with the more rigorous consistency condition (Eq. (15)), for the three field models.

\subsection{Ring model}

In the ring model, $\left\langle B_{r}\right\rangle$ vanishes and $\left\langle B_{\theta}\right\rangle$ is constant along circles. Hence, there are 9 free parameters: $\left\langle B_{\theta}\right\rangle_{i}$, the large-scale 
Table 1. Critical values of $\chi^{2}$ for 3 probability levels.

\begin{tabular}{cccc}
\hline \hline$n_{i}-v$ & $\chi_{\text {crit }}^{2}\left(P_{0}=0.05\right)$ & $\chi_{\text {crit }}^{2}\left(P_{0}=0.1\right)$ & $\chi_{\text {crit }}^{2}\left(P_{0}=0.2\right)$ \\
\hline 1 & 3.841 & 2.706 & 1.642 \\
2 & 5.991 & 4.605 & 3.219 \\
3 & 7.815 & 6.251 & 4.642 \\
4 & 9.488 & 7.779 & 5.989 \\
5 & 11.070 & 9.236 & 7.289 \\
\hline
\end{tabular}

azimuthal fields in the $9 \operatorname{rings} i=4,4.5,5 \ldots 8^{1}$. Since all the free parameters are independent, the 9 rings can be treated separately.

For every ring $i$, the large-scale line-of-sight field in any box $j$ is simply the projection of $\left\langle B_{\theta}\right\rangle_{i}$ onto the line of sight (see Eq. (5) with $\left\langle B_{r}\right\rangle=0$ ):

$\left\langle B_{\|}\right\rangle_{i j}=\left\langle B_{\theta}\right\rangle_{i} \cos \alpha_{i j}$,

where $\alpha_{i j}$ is the angle between the azimuthal direction and the direction to the Sun at the midpoint ${ }^{2}$ of box $j$ (see Fig. 1). The best-fit value of $\left\langle B_{\theta}\right\rangle_{i}$ is obtained by minimizing $\chi^{2}$ (given by Eq. (13)). In terms of $\left\langle\overline{B_{\theta}}\right\rangle_{i j}=\left\langle\overline{B_{\|}}\right\rangle_{i j} / \cos \alpha_{i j}$, the observational value of the average azimuthal field in box $j$, and $\left(\sigma_{\theta}\right)_{i j}=\left(\sigma_{\|}\right)_{i j} / \cos \alpha_{i j}$, the associated uncertainty, the minimization procedure turns out to be equivalent to taking an uncertainty-weighted average of the different $\left\langle\overline{B_{\theta}}\right\rangle_{i j}$ along ring $i$ :

$$
\left\langle B_{\theta}\right\rangle_{i}=\frac{\sum_{j=1}^{n_{i}} \frac{\left\langle\overline{B_{\theta}}\right\rangle_{i j}}{\left(\sigma_{\theta}\right)_{i j}^{2}}}{\sum_{j=1}^{n_{i}} \frac{1}{\left(\sigma_{\theta}\right)_{i j}^{2}}} .
$$

The values of $\left\langle\overline{B_{\theta}}\right\rangle_{i j}$ and their uncertainties $\left(\sigma_{\theta}\right)_{i j}$ in the $n_{i}$ boxes $j$ of the 9 rings $i$ are plotted in Fig. 5, at the Galactic azimuthal angles of the box midpoints, $\theta_{i j}$. For comparison, the best-fit values of $\left\langle B_{\theta}\right\rangle_{i}$ in the 9 rings are indicated by horizontal lines spanning the entire azimuthal range.

In only one ring $(i=4.5)$ does the best-fit value of $\left\langle B_{\theta}\right\rangle_{i}$ satisfy the rule-of-thumb consistency condition, $\chi^{2} \leq n_{i}-1$ (Eq. (14) with $v=1$ ). For this ring, we compute the consistency range of $\left\langle B_{\theta}\right\rangle_{i}$, which contains all the values of $\left\langle B_{\theta}\right\rangle_{i}$ for which $\chi^{2} \leq n_{i}-1$. The best-fit value of $\left\langle B_{\theta}\right\rangle_{i}$ and its consistency range in the sole "good-fit" ring are plotted against $r_{i}$, in the upper panel of Fig. 6. For the other 8 rings, the (inconsistent) best-fit values of $\left\langle B_{\theta}\right\rangle_{i}$ are plotted with crosses. Clearly, these 8 rings do not admit any ring magnetic field consistent with the data. As an immediate consequence, the ring model must be rejected.

In order to gain some feel for how far the ring model is from being able to reproduce the pulsar data, let us, in thought, extend the error bars of all the observational $\left\langle\overline{B_{\|}}\right\rangle_{i j}$ by a factor of 2 and look into the impact of this extension on our results. With twice the original error bars, the $\chi^{2}$ parameter would be smaller by a factor of 4 , so that, in terms of the original $\chi^{2}$, the rule-of-thumb consistency condition would become $\chi^{2} \leq 4\left(n_{i}-1\right)$. As it turns out, this less stringent consistency condition would be fulfilled

\footnotetext{
1 As a reminder, ring $i$ is centered on $r_{i} \equiv i \mathrm{kpc}$ and extends between $(i-0.5) \mathrm{kpc}$ and $(i+0.5) \mathrm{kpc}$.

2 As in Fig. 4, the midpoint of a box is defined as the point at middle radius, $r=r_{i}$, and middle longitude, $l=\left(l_{\min }+l_{\max }\right) / 2$.
}

ring 4

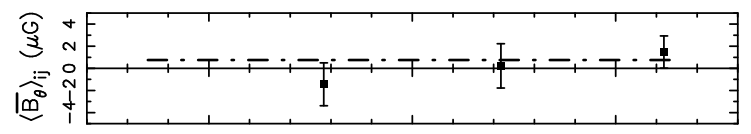

ring 4.5

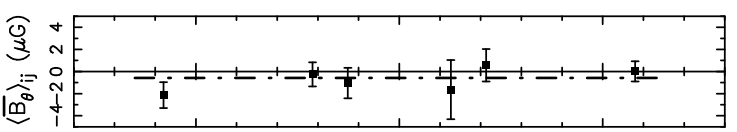

ring 5



ring 5.5

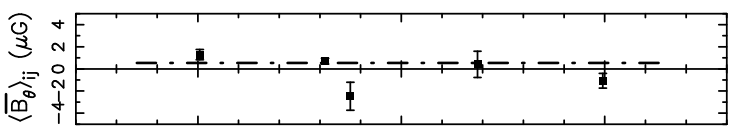

ring 6

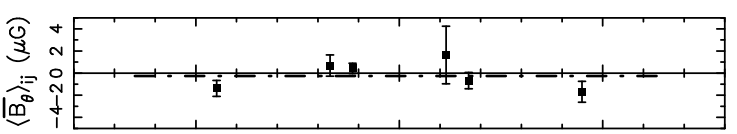

ring 6.5

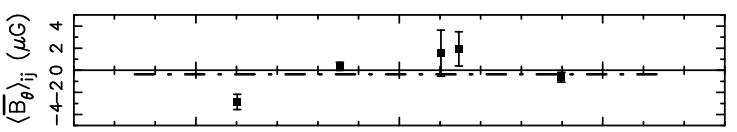

ring 7

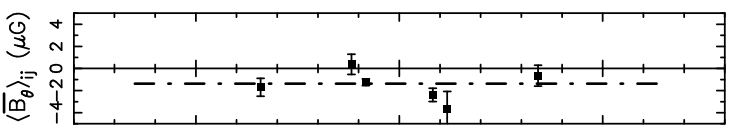

ring 7.5

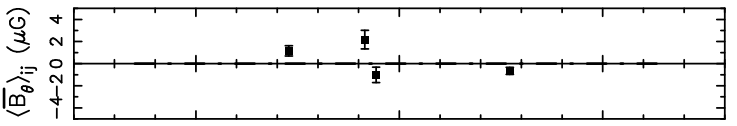

ring 8

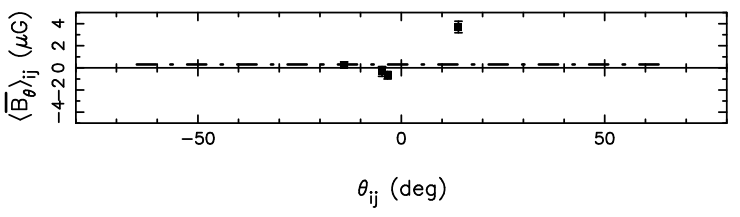

Fig. 5. Observational values of the average azimuthal fields, $\left\langle\overline{B_{\theta}}\right\rangle_{i j}$, versus Galactic azimuthal angles, $\theta_{i j}$, in all the boxes $j$ of the 9 successive rings $i$, for the ring model. The uncertainties $\left(\sigma_{\theta}\right)_{i j}$ in the field values are plotted as standard error bars. For each ring $i$, the best-fit value of the model parameter $\left\langle B_{\theta}\right\rangle_{i}$ is indicated by the horizontal dot-dashed line.

in 5 rings $(i=4,4.5,5.5,6,7)$ out of 9 . Thus, with twice the original error bars, the ring model would remain unacceptable.

If we now resort to the more rigorous consistency condition, $\chi^{2} \leq \chi_{\text {crit }}^{2}(0.1)$ (Eq. (15) with $\left.P_{0}=0.1\right)$, to test the ring model, we find that 3 rings $(i=4,4.5,6)$ have their best-fit $\left\langle B_{\theta}\right\rangle_{i}$ consistent with the data; their consistency ranges are drawn in the lower panel of Fig. 6. For the other 6 rings, the (inconsistent) best-fit $\left\langle B_{\theta}\right\rangle_{i}$ are again plotted with crosses. With $P_{0}=0.05$, 4 rings $(i=4,4.5,6,7)$ would be deemed consistent with the data, but the other 5 rings would still fail the consistency test.

These results confirm our conclusion that the ring model must be rejected.

\subsection{Axisymmetric model}

In the axisymmetric model, $\left\langle B_{r}\right\rangle$ and $\left\langle B_{\theta}\right\rangle$ are both constant along circles. Hence, there are 18 free parameters: $\left\langle B_{r}\right\rangle_{i}$ and $\left\langle B_{\theta}\right\rangle_{i}$, the large-scale radial and azimuthal fields in the 9 rings $i=4,4.5,5 \ldots 8$. 

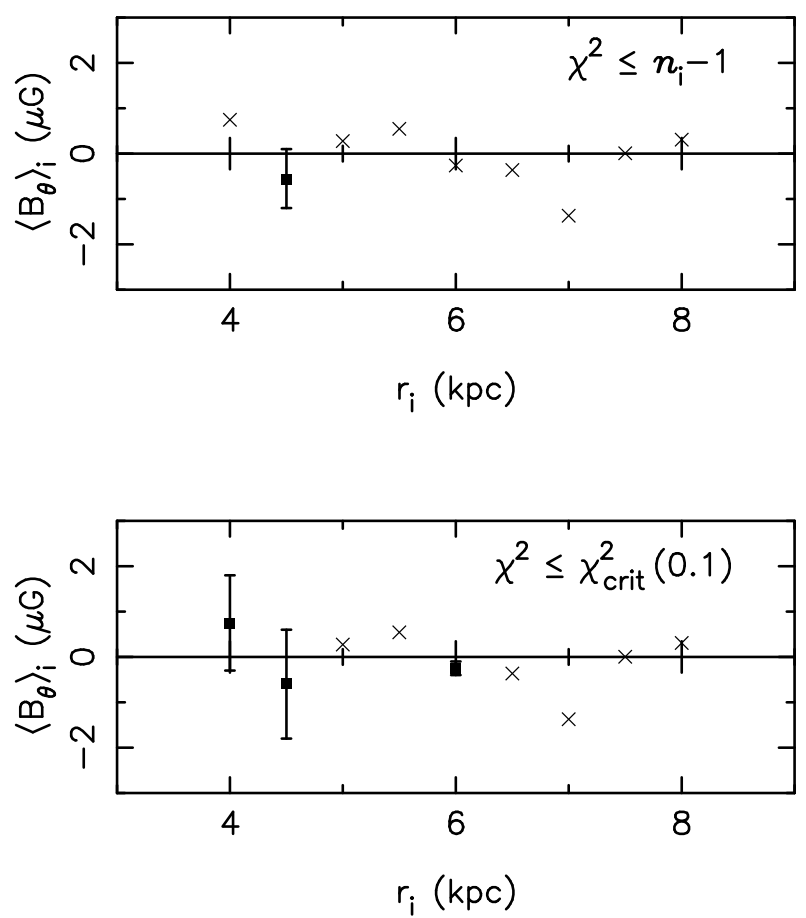

Fig. 6. Best-fit values of the model parameters, $\left\langle B_{\theta}\right\rangle_{i}$, versus ring middle radii, $r_{i}$, for the ring model. The acceptable values (those consistent with the pulsar data) are shown with their consistency ranges, while the unacceptable values are indicated with crosses. The results obtained with the rule-of-thumb consistency condition, $\chi^{2} \leq n_{i}-1$, are plotted in the upper panel. Those obtained with the more rigorous consistency condition, $\chi^{2} \leq \chi_{\text {crit }}^{2}(0.1)$, are plotted in the lower panel.

For every ring $i$, the large-scale line-of-sight field in any box $j$ can be written as a linear combination of the two parameters $\left\langle B_{r}\right\rangle_{i}$ and $\left\langle B_{\theta}\right\rangle_{i}$ :

$$
\left\langle B_{\|}\right\rangle_{i j}=\left\langle B_{r}\right\rangle_{i} \sin \alpha_{i j}+\left\langle B_{\theta}\right\rangle_{i} \cos \alpha_{i j}
$$

(see Eq. (5)). Again the best-fit values of $\left\langle B_{r}\right\rangle_{i}$ and $\left\langle B_{\theta}\right\rangle_{i}$ are obtained by minimizing $\chi^{2}$ (Eq. (13)).

Here, we find that 1 ring $(i=4.5)$ has its best fit consistent with the data, according to the rule-of-thumb consistency condition, $\chi^{2} \leq n_{i}-2$ (Eq. (14) with $v=2$ ). Its consistency domain in the parameter plane $\left(\left\langle B_{r}\right\rangle_{i},\left\langle B_{\theta}\right\rangle_{i}\right)$ is the area delimited by the ellipse $\chi^{2}=n_{i}-2$ (grey contour line in the relevant panel of Fig. 7). As none of the other 8 rings can be properly fit with an axisymmetric magnetic field, the axisymmetric model must be rejected.

With twice the original error bars on the observational $\left\langle\overline{B_{\|}}\right\rangle_{i j}$, the rule-of-thumb consistency condition would become $\chi^{2} \leq$ $4\left(n_{i}-2\right)$ (in terms of the original $\left.\chi^{2}\right)$. This less stringent consistency condition would be satisfied in 5 rings $(i=4,4.5,6,7,8)$, but still not in the other 4 rings. Therefore, the axisymmetric model would remain unacceptable.

According to the more rigorous consistency condition, $\chi^{2} \leq$ $\chi_{\text {crit }}^{2}(0.1)\left(\right.$ Eq. (15) with $\left.P_{0}=0.1\right), 5$ rings $(i=4,4.5,6,7,8)$ have their best fits consistent with the data. Their consistency domains in the parameter planes $\left(\left\langle B_{r}\right\rangle_{i},\left\langle B_{\theta}\right\rangle_{i}\right)$ are the elliptical areas enclosed by the curves $\chi^{2}=\chi_{\text {crit }}^{2}(0.1)$ (black contour lines in the relevant panels of Fig. 7). For the other 4 rings, the (inconsistent) best-fit pairs $\left(\left\langle B_{r}\right\rangle_{i},\left\langle B_{\theta}\right\rangle_{i}\right)$ are indicated with crosses. Relaxing the probability level to $P_{0}=0.05$ would not raise the number of acceptable rings above 5 .
From all the above, we conclude that the axisymmetric model must be rejected.

\subsection{Bisymmetric model}

In the bisymmetric model, $\left\langle B_{r}\right\rangle$ and $\left\langle B_{\theta}\right\rangle$ vary sinusoidally along circles in the manner described by Eqs. (10) and (11). Hence, there are 27 free parameters: $b_{r, i}, b_{\theta, i}$ and $\phi_{i}$, the maximum amplitudes and the azimuthal phases in the 9 rings $i=4,4.5,5 \ldots 8$.

For every ring $i$, the large-scale line-of-sight field in any box $j$ follows from Eq. (5) together with Eqs. (10), (11):

$\left\langle B_{\|}\right\rangle_{i j}=b_{r, i} \sin \left(\theta_{i j}-\phi_{i}\right) \sin \alpha_{i j}+b_{\theta, i} \sin \left(\theta_{i j}-\phi_{i}\right) \cos \alpha_{i j}$,

where the angles $\theta_{i j}$ and $\alpha_{i j}$ (see Fig. 1) refer to the midpoint of box $j$. Similarly to the previous models, the best-fit values of the three parameters $b_{r, i}, b_{\theta, i}$ and $\phi_{i}$ are obtained through a minimization of $\chi^{2}$ (Eq. (13)).

According to the rule-of-thumb consistency condition, $\chi^{2} \leq$ $n_{i}-3$ (Eq. (14) with $v=3$ ), 2 rings $(i=4.5,5)$ have their best fits consistent with the data. Their consistency domains in the parameter spaces $\left(b_{r, i}, b_{\theta, i}, \phi_{i}\right)$ are the volumes bounded by the surfaces $\chi^{2}=n_{i}-3$. Displayed in Fig. 8 are the projections of these consistency domains on the parameter planes $\left(b_{r, i}, b_{\theta, i}\right)$ (grey contour lines). Since the other 7 rings fail the consistency test, the bisymmetric model must be rejected.

With twice the original error bars on the observational $\left\langle\overline{B_{\|}}\right\rangle_{i j}$, the rule-of-thumb consistency condition would become $\chi^{2} \leq 4\left(n_{i}-3\right)$, which would be satisfied in $7 \operatorname{rings}(i=$ $4,4.5,5,5.5,6,7,8)$ out of 9 . The bisymmetric model would then be nearly acceptable.

According to the more rigorous consistency condition, $\chi^{2} \leq$ $\chi_{\text {crit }}^{2}(0.1)\left(\right.$ Eq. (15) with $\left.P_{0}=0.1\right), 5$ rings $(i=4.5,5,6,7,8)$ have their best fits consistent with the data. Their consistency domains, bounded by the surfaces $\chi^{2}=\chi_{\text {crit }}^{2}(0.1)$, are also shown in projection on the parameter planes $\left(b_{r, i}, b_{\theta, i}\right)$ in Fig. 8 (black contour lines). For the other 4 rings, the (inconsistent) best-fit pairs $\left(b_{r, i}, b_{\theta, i}\right)$ are indicated with crosses. Relaxing the probability level to $P_{0}=0.05$ would raise the number of acceptable rings to $7(i=4,4.5,5,5.5,6,7,8)$, which would render the bisymmetric model nearly globally acceptable.

Altogether, the bisymmetric model must be rejected, though its rejection is slightly less severe than for the axisymmetric model.

\section{Summary and conclusions}

In this paper, we examined the three most common theoretical models for the large-scale magnetic field in the Galactic disk and confronted each of these models with the pulsar data. For each model, we derived the best-fit parameters, through $\chi^{2}$ minimization, in the 9 Galactocentric rings defined in Fig. 2, and we delineated the parameter domains around the best fits (referred to as the consistency domains) wherein the predicted fields are consistent with the pulsar data. 


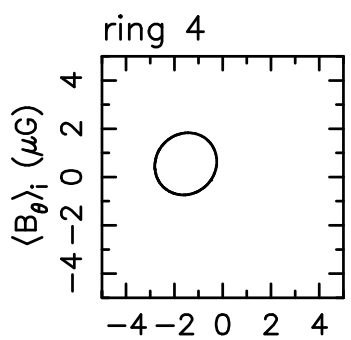

$\left\langle\mathrm{B}_{\mathrm{r}}\right\rangle_{\mathrm{i}}(\mu \mathrm{G})$

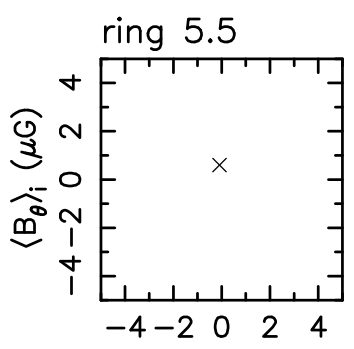

$\left\langle\mathrm{B}_{\mathrm{r}}\right\rangle_{\mathrm{i}}(\mu \mathrm{G})$

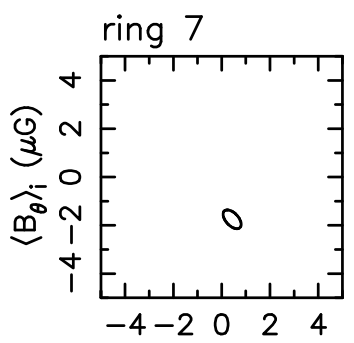

$\left\langle\mathrm{B}_{\mathrm{r}}\right\rangle_{\mathrm{i}}(\mu \mathrm{G})$

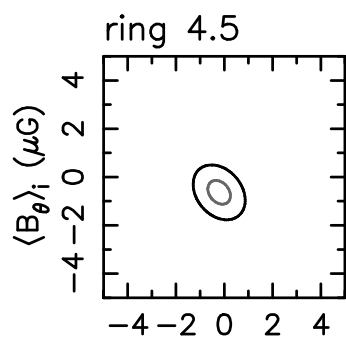

$\left\langle B_{r}\right\rangle_{i}(\mu G)$

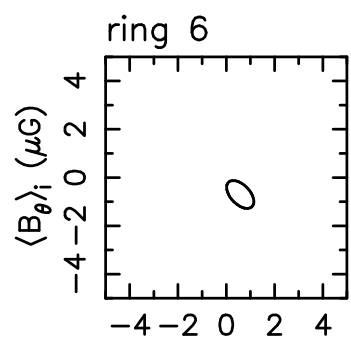

$\left\langle\mathrm{B}_{\mathrm{r}}\right\rangle_{\mathrm{i}}(\mu \mathrm{G})$

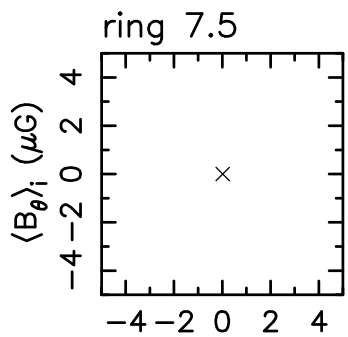

$\left\langle\mathrm{B}_{\mathrm{r}}\right\rangle_{\mathrm{i}}(\mu \mathrm{G})$

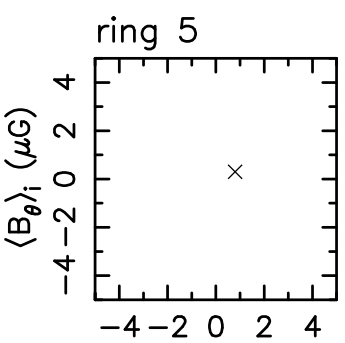

$\left\langle\mathrm{B}_{\mathrm{r}}\right\rangle_{\mathrm{i}}(\mu \mathrm{G})$

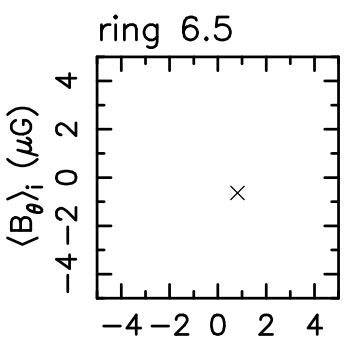

$\left\langle\mathrm{B}_{\mathrm{r}}\right\rangle_{\mathrm{i}}(\mu \mathrm{G})$

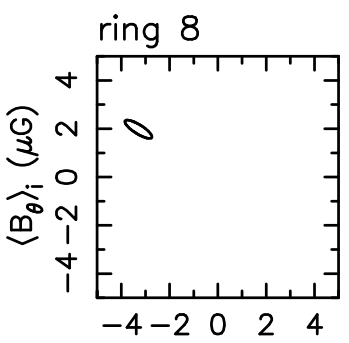

$\left\langle\mathrm{B}_{\mathrm{r}}\right\rangle_{\mathrm{i}}(\mu \mathrm{G})$

Fig. 7. Consistency domains in the parameter planes $\left(\left\langle B_{r}\right\rangle_{i},\left\langle B_{\theta}\right\rangle_{i}\right)$ of the 9 different rings, for the axisymmetric model. The grey contour lines define the consistency domains obtained with the rule-of-thumb consistency condition, $\chi^{2} \leq n_{i}-2$. The black contour lines define those obtained with the more rigorous consistency condition, $\chi^{2} \leq \chi_{\text {crit }}^{2}(0.1)$. The crosses mark the locations of the unacceptable best fits (those inconsistent with the pulsar data according to both criteria).

Compared to existing studies of the kind, we did not attempt to settle the long-standing (and possibly ill-posed) question of whether the Galactic magnetic field is axisymmetric or bisymmetric. Our sole purpose was to determine whether each of the three basic field models, taken separately, is compatible with the available pulsar data or not. In this regard, we note that many previous studies did find a preference for one of the field models, but omitted to put their preferred model through the crucial "goodness-of-fit" test, which checks whether the model can indeed reproduce the data within the error bars.

Here, we tested the three field models on the basis of two different criteria: first, a standard rule of thumb for a reasonably good fit (Eq. (14)), and second, a more rigorous consistency condition for a chi-square distribution of $\chi^{2}$ (Eq. (15), with the imposed probability set to $P_{0}=0.1$ ). These two criteria were successively applied to all the rings separately, such that the best fit of ring $i$ was deemed consistent with the pulsar data if the associated value of $\chi^{2}, \chi_{\min }^{2}$, was less than $n_{i}-v$ (first criterion) or less than $\chi_{\text {crit }}^{2}\left(P_{0}=0.1\right)$ (second criterion). A model could then be considered globally acceptable if all the rings had their best fits consistent with the data.
The results obtained for the three field models, with both criteria, are summarized in Table 2. All the rings are listed with their labels, $i$ (see footnote 1), and their numbers of boxes (or numbers of data points), $n_{i}$, from which it is straightforward to deduce the numbers of degrees of freedom, $n_{i}-v$ (for the first criterion), and the critical $\chi_{\text {crit }}^{2}\left(P_{0}=0.1\right)$ (for the second criterion; see Table 1). Also given for all the rings are the minimum values of $\chi^{2}, \chi_{\min }^{2}$, i.e., the values associated with the best fits, as well as the results of both consistency tests (satisfaction of a test is indicated with an asterisk), for the three field models.

We found that none of the three field models is acceptable, in the sense that none of them can be brought into full agreement with the pulsar data. According to the standard rule-ofthumb consistency condition (Eq. (14)), all three models must be strongly rejected, as the ring and axisymmetric models fail to provide a good fit (consistent with the data) in all the rings save one, while the bisymmetric model fails in all the rings save two. If the error bars of the observational line-of-sight fields were enlarged by a factor of 2 , the bisymmetric model would not be too far from acceptable ( 7 good-fit rings out of 9), while the ring and axisymmetric models would remain truly unacceptable (5 goodfit rings out of 9). The conclusions reached with the more 

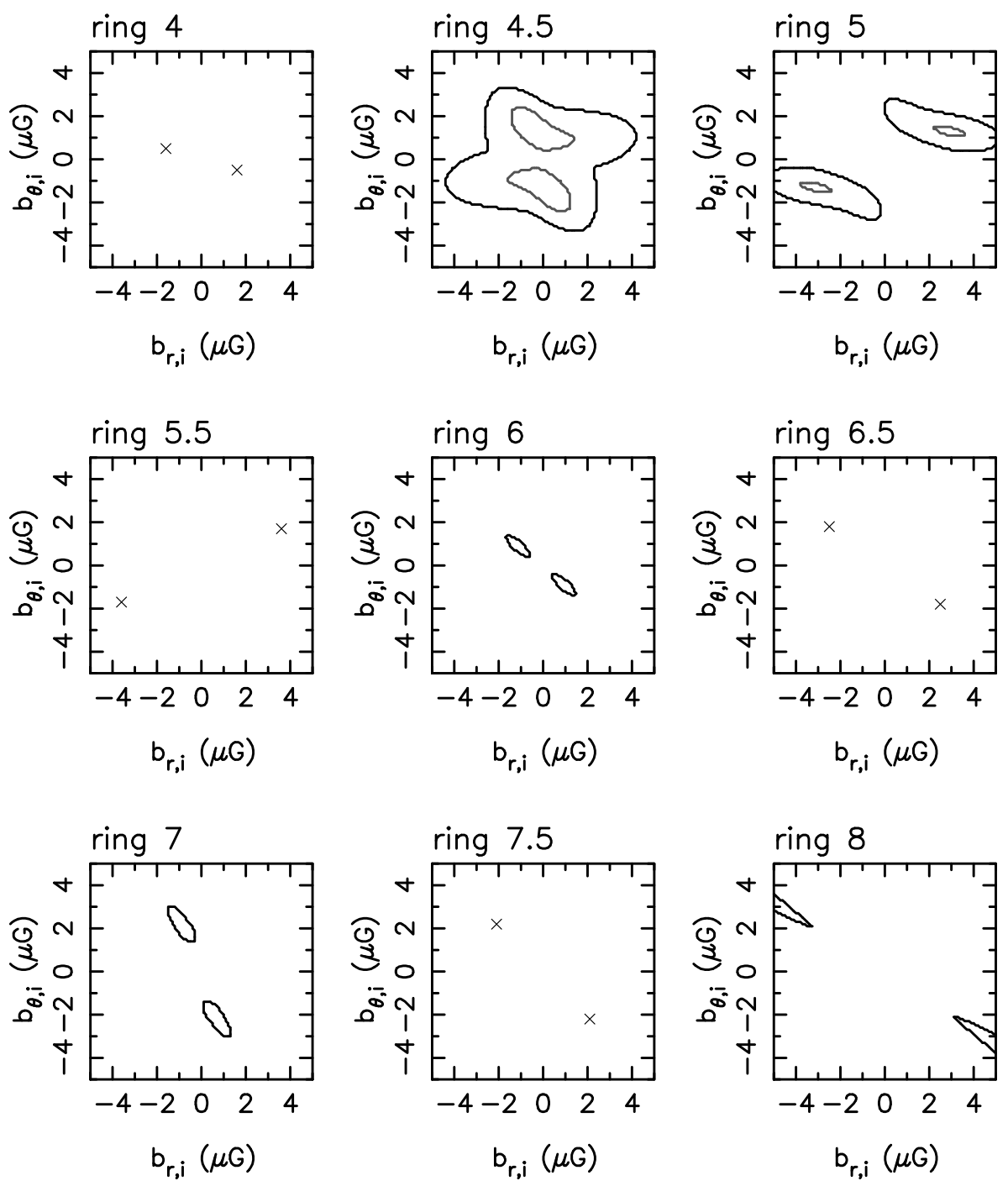

Fig. 8. Projections of the 3D consistency domains of the 9 different rings on their parameter planes $\left(b_{r, i}, b_{\theta, i}\right)$, for the bisymmetric model. The grey contour lines correspond to the consistency domains obtained with the rule-of-thumb consistency condition, $\chi^{2} \leq n_{i}-3$. The black contour lines correspond to those obtained with the more rigorous consistency condition, $\chi^{2} \leq \chi_{\text {crit }}^{2}(0.1)$. The crosses mark the locations of the unacceptable best fits (those inconsistent with the pulsar data according to both criteria).

rigorous consistency condition (Eq. (15)) are intermediate between the two situations described above: with the imposed probability set to $P_{0}=0.1\left(P_{0}=0.05\right), 3$ (4) rings can be properly fit with a ring magnetic field, 5 (5) with an axisymmetric field and 5 (7) with a bisymmetric field.

The quantitative differences between both criteria are easily understood. A comparison between Eqs. (14) and (15) immediately shows that the rule-of-thumb consistency condition (Eq. (14)) corresponds to a probability level $P_{1}$ such that $\chi_{\text {crit }}^{2}\left(P_{1}\right)=n_{i}-v$, or equivalently, $P_{1}=P\left[\chi^{2}>n_{i}-v\right]$. With twice the original error bars on the observational line-of-sight fields, the rule-of-thumb consistency condition would become $\chi^{2} \leq 4\left(n_{i}-v\right)$, corresponding to a probability level $P_{2}$ such that $\chi_{\text {crit }}^{2}\left(P_{2}\right)=4\left(n_{i}-v\right)$, or equivalently, $P_{2}=P\left[\chi^{2}>4\left(n_{i}-v\right)\right]$. The values of $P_{1}$ and $P_{2}$ as functions of $n_{i}-v$ are tabulated in Table 3. Clearly, the rule-of-thumb consistency condition with the original error bars implies high probability levels $\left(P_{1} \sim 30 \%-40 \%\right)$, which make it overly difficult to satisfy; if the model under testing is correct, there is nonetheless a $\sim 30 \%-40 \%$ chance that $\chi^{2}$ exceeds $n_{i}-v$ and that the model will be rejected. On the other hand, with twice the original error bars, the probability levels drop very low $\left(P_{2} \sim 0.1 \%-4 \%\right)$ and the consistency condition becomes too easily satisfied; the risk is then to accept a model that is in fact incorrect.

The bottom line is that the standard rule of thumb is way too stringent, while the rule of thumb with twice the original error bars is way too easy. In contrast, the more rigorous criterion with a probability level set to $P_{0}=0.1$, intermediate between $P_{1}$ and $P_{2}$, provides a reasonable trade-off. The latter criterion is also more trustworthy, insofar as all the rings for all the models are tested with the same probability level.

The results of the present study suggest that the true largescale magnetic field in our Galaxy has a more complex configuration than a strictly axisymmetric or bisymmetric field. One possibility is that it consists of the superposition of axisymmetric, bisymmetric and probably higher-order azimuthal modes. Such combinations of modes have been observed in a number of external galaxies (e.g., Beck et al. 1996; Berkhuijsen et al. 1997; Rohde et al. 1999; Beck 2007). 
Table 2. Summary of the results obtained for the three field models ${ }^{a}$.

\begin{tabular}{|c|c|c|c|c|c|c|c|c|c|c|}
\hline & & \multicolumn{3}{|c|}{ Ring model $(v=1)$} & \multicolumn{3}{|c|}{ Axisymmetric model $(v=2)$} & \multicolumn{3}{|c|}{ Bisymmetric model $(v=3)$} \\
\hline $\bar{i}$ & $n_{i}$ & $\chi_{\min }^{2}$ & {$\left[\chi_{\min }^{2} \leq n_{i}-v\right]$} & {$\left[\chi_{\min }^{2} \leq \chi_{\text {crit }}^{2}(0.1)\right]$} & $\chi_{\min }^{2}$ & {$\left[\chi_{\min }^{2} \leq n_{i}-v\right]$} & {$\left[\chi_{\min }^{2} \leq \chi_{\text {crit }}^{2}(0.1)\right]$} & $\overline{\chi_{\min }^{2}}$ & {$\left[\chi_{\min }^{2} \leq n_{i}-v\right]$} & {$\left[\chi_{\min }^{2} \leq \chi_{\text {crit }}^{2}(0.1)\right]$} \\
\hline 4 & 4 & 5.03 & & $*$ & 2.91 & & $*$ & 3.08 & & \\
\hline 4.5 & 6 & 3.17 & $*$ & $*$ & 3.11 & * & * & 1.75 & * & * \\
\hline 5 & 5 & 25.37 & & & 18.65 & & & 1.79 & * & $*$ \\
\hline 5.5 & 5 & 14.52 & & & 14.43 & & & 4.95 & & \\
\hline 6 & 6 & 8.84 & & $*$ & 5.06 & & $*$ & 5.48 & & $*$ \\
\hline 6.5 & 5 & 20.29 & & & 17.93 & & & 13.55 & & \\
\hline 7 & 6 & 9.57 & & & 5.81 & & $*$ & 3.37 & & $*$ \\
\hline 7.5 & 4 & 19.31 & & & 19.30 & & & 7.44 & & \\
\hline 8 & 4 & 52.53 & & & 3.04 & & $*$ & 0.82 & & $*$ \\
\hline
\end{tabular}

a When a value of $\chi_{\min }^{2}$ satisfies the first or second consistency condition, an asterisk is plotted in the corresponding column.

Table 3. Probability levels for the rule-of-thumb consistency condition.

\begin{tabular}{ccc}
\hline \hline$n_{i}-v$ & $P_{1}=P\left[\chi^{2}>n_{i}-v\right]$ & $P_{2}=P\left[\chi^{2}>4\left(n_{i}-v\right)\right]$ \\
\hline 1 & 0.3173 & 0.0455 \\
2 & 0.3679 & 0.0183 \\
3 & 0.3916 & 0.0074 \\
4 & 0.4060 & 0.0030 \\
5 & 0.4159 & 0.0012 \\
\hline
\end{tabular}

Acknowledgements. We thank the referee for his/her valuable comments as well as Pierre Jean and William Gillard for useful discussions. J.L.H. and M.H. were supported by the National Natural Science Foundation (NNSF) of China (10521001 and 10773016) and the National Key Basic Research Science Foundation of China (2007CB815403). M.H. was also supported by the French Embassy in Beijing during her stay in Toulouse in the framework of a bilateral co-supervised Ph.D. program.

\section{References}

Beck, R. 2007, A\&A 470, 539

Beck, R., Brandenburg, A., Moss, D., et al. 1996, ARA\&A, 34, 155 Beck, R., Shukurov, A., Sokoloff, D., \& Wielebinski, R. 2003, A\&A, 411, 99 Berkhuijsen, E. M., Horellou, C., Krause, M., et al. 1997, A\&A, 318, 700 Cordes, J. M., \& Lazio, T. J. W. 2002 [arXiv: astro-ph/0207156v3] Crawford, F., Manchester, R. N., \& Kaspi, V. M. 2001, AJ, 122, 2001 Ferrière, K., \& Schmitt, D. 2000, A\&A, 358, 125
Hamilton, P. A., \& Lyne, A. G. 1987, MNRAS, 224, 1073

Han, J. L., \& Qiao, G. J. 1994, A\&A, 288, 759

Han, J. L., Manchester, R. N., \& Qiao, G. J. 1999, MNRAS, 306, 37

Han, J. L., Manchester, R. N., Lyne, A. G., et al. 2006, ApJ, 642, 868 Howard, A. M., \& Kulsrud, R. M. 1997, ApJ, 483, 648

Indrani, C., \& Deshpande, A. A. 1999, NewA, 4, 33

Isobe, T., Feigelson, E. D., Akritas, M. G., et al. 1990, ApJ, 364, 104

Manchester, R. N., Hobbs, G. B., Teoh, A., et al. 2005, AJ, 129, 1993

Mitra, D., Wielebinski, R., Kramer, M., et al. 2003, A\&A, 398, 993

Moss, D. 1995, MNRAS, 275, 191

Moss, D. 1996, A\&A, 315, 63

Noutsos, A., Johnston, S., Kramer, M., \& Karastergiou, A. 2008 [arXiv: 0803.0677]

Poezd, A., Shukurov, A., \& Sokoloff, D. 1993, MNRAS, 264, 285

Press, W. H., Teukolsky, S. A., Vetterling, W. T., \& Flannery, B. P. 1992, Numerical Recipes FORTRAN: the Art of Scientific Computing, 2nd edn. (Cambridge: Cambridge Univ. Press)

Qiao, G. J., Manchester, R. N., Lyne, A. G., et al. 1995, MNRAS, 274, 572

Rand, R. J., \& Kulkarni, S. R. 1989, ApJ, 343, 760

Rand, R. J., \& Lyne, A. G. 1994, MNRAS, 268, 497

Rohde, R., Beck, R., \& Elstner, D. 1999, A\&A, 350, 423

Ruzmaikin, A. A., Sokolov, D. D., \& Shukurov, A. M. 1985, A\&A, 148, 335

Simard-Normandin, M., \& Kronberg, P. P. 1980, ApJ, 242, 74

Vallée, J. P. 1991, ApJ, 366, 450

Vallée, J. P. 1996, A\&A, 308, 433

Vallée, J. P. 2005, ApJ, 619, 297

van Ommen, T. D., D'Alesssandro, F. D., Hamilton, P. A., et al. 1997, MNRAS, 287, 307

Weisberg, J. M., Cordes, J. M., Kuan, B., et al. 2004, ApJS, 150, 317

Yamane, T. 1964, Statistics, an introductory Analysis (New York: Harper \& Row) 